\title{
Inhibition of Protein Tyrosine Phosphatase Improves Angiogenesis via Enhancing Ang-1/Tie-2 Signaling in Diabetes
}

\author{
Jian-Xiong Chen, ${ }^{1}$ Qinhui Tuo, ${ }^{2}$ Duan-Fang Liao, ${ }^{3}$ and Heng Zeng ${ }^{1}$ \\ ${ }^{1}$ Department of Pharmacology and Toxicology, University of Mississippi Medical Center, 2500 North State Street, \\ Jackson, MS 39216, USA \\ ${ }^{2}$ Department of Pharmacology, University of South of China, Hengyang 421001, China \\ ${ }^{3}$ Department of Traditional Chinese Diagnostics, School of Pharmacy, Hunan University of Chinese Medicine, \\ Hunan, Changsha 410208, China \\ Correspondence should be addressed to Jian-Xiong Chen, jchen3@umc.edu
}

Received 8 June 2011; Revised 12 November 2011; Accepted 13 November 2011

Academic Editor: Hiroshi Okamoto

Copyright (c) 2012 Jian-Xiong Chen et al. This is an open access article distributed under the Creative Commons Attribution License, which permits unrestricted use, distribution, and reproduction in any medium, provided the original work is properly cited.

\begin{abstract}
Diabetes is associated with impairment of angiogenesis such as reduction of myocardial capillary formation. Our previous studies demonstrate that disruption of Angiopoietin-1 (Ang-1)/Tie-2 signaling pathway contributes to the diabetes-associated impairment of angiogenesis. Protein tyrosine phosphatase (PTP) has a critical role in the regulation of insulin signal by inhibition of tyrosine kinase phosphorylation. In present study, we examined the role of protein tyrosine phosphatase-1 (SHP-1) in diabetes-associated impairment of Ang-1/Tie-2 angiogenic signaling and angiogenesis. SHP-1 expression was significantly increased in diabetic db/db mouse hearts. Furthermore, SHP-1 bond to Tie-2 receptor and stimulation with Ang-1 led to SHP-1 dissociation from Tie-2 in mouse heart microvascular endothelial cell (MHMEC). Exposure of MHMEC to high glucose (HG, $30 \mathrm{mmol} / \mathrm{L}$ ) increased SHP1/Tie-2 association accompanied by a significant reduction of Tie-2 phosphorylation. Exposure of MHMEC to HG also blunted Ang-1-mediated SHP-1/Tie-2 dissociation. Knockdown of SHP-1 significantly attenuated HG-induced caspase-3 activation and apoptosis in MHMEC. Treatment with PTP inhibitors restored Ang-1-induced Akt/eNOS phosphorylation and angiogenesis. Our data implicate a critical role of SHP-1 in diabetes-associated vascular complications, and that upregulation of Ang-1/Tie-2 signaling by targeting SHP-1 should be considered as a new therapeutic strategy for the treatment of diabetes-associated impairment of angiogenesis.
\end{abstract}

\section{Introduction}

Angiogenesis is mainly regulated by the vascular endothelial growth factor (VEGF)/VEGF receptor (VEGFR) and the angiopoietins/Tie-2 system. Receptor tyrosine kinases (RTKs) represent a major class of cell-surface molecules that regulate angiogenesis. VEGFR and the Tie-2 receptor are the principal RTK families and play critical roles in the regulation of angiogenesis [1]. Impaired angiogenesis leading to microvascular insufficiency represents a major cause of end-stage organ failure among diabetics. The underlying molecular mechanisms, however, are poorly understood $[2,3]$. Myocardial angiogenesis is significantly impaired in patients with diabetes mellitus which may contribute to the high mortality after myocardial infarction $[4,5]$. So far, few studies have focused on the identification of factors that affect myocardial angiogenesis in the setting of diabetes. A previous study showed that VEGF-induced migration and VEGFR-mediated signal transduction were severely impaired in the monocytes of diabetic patients $[6,7]$. Further, VEGFR expression was significantly reduced in the heart of diabetic patients compared with nondiabetic individuals. This was accompanied by an impairment of VEGFR phosphorylation, suggesting that decreased VEGF expression and defective VEGF signaling may play a key role in the diabetes-associated impairment of angiogenesis [8]. Our previous studies have found that defective RTK signaling transduction is not only limited to VEGF/VEGFR, but is also associated with 
the disruption of Ang-1/Tie-2 angiogenic signaling and angiogenesis under hyperglycemic conditions and in diabetes [9-11].

Protein tyrosine phosphatase (PTP) has been shown to negatively regulate insulin signaling by dephosphorylation of insulin receptor tyrosine kinase $[12,13]$. PTP also has a critical role in the regulation of growth factors signal transduction by de-phosphorylation of RTK. PTP inhibition has been shown to promote collateral growth and enhance VEGF-induced angiogenesis in a rat model of hindlimb ischemia $[14,15]$. The cytoplasmic protein tyrosine phosphatase-1 (SHP-1) expresses primarily in hematopoietic lineages and endothelial cells [16-19] and negatively regulates growth factor receptors phosphorylation $[17,18,20$, 21]. SHP-1 expression is upregulated as a result of abnormal inflammatory responses in diabetes patients [22]. A previous study revealed that Tie-2 receptor was the substrates for tyrosine phosphatase-2 (SHP-2) [23]. To date, little is known of the functional role of SHP- 1 on the Ang- $1 /$ Tie-2 signaling and impairment of angiogenesis in diabetes.

In our present study, we hypothesize that hyperglycemia and diabetes impair Ang-1/Tie-2 signaling and angiogenesis by a mechanism involving upregulation of SHP-1 expression and SHP-1/Tie-2 interaction. Our data suggest that increased SHP-1 has a crucial role in the diabetes-associated impairment of angiogenesis by interfering with the Ang- $1 /$ Tie- 2 angiogenic signaling.

\section{Materials and Methods}

2.1. Mouse Heart Microvascular Endothelial Cells (MHMECs). MHMECs was isolated from C57BL/6J mouse hearts and cultured as previously described [24-26]. Primary cultures of MHMEC, between passages 4 and 10, were used in all experiments.

2.2. Endothelial Cell Apoptosis and Caspase-3 Activity. To induce apoptosis, MHMEC were exposed to serum-free medium for 72 hours under high glucose (HG, $30 \mathrm{mmol} / \mathrm{L}$ ) or normal glucose (NG, $5 \mathrm{mmol} / \mathrm{L}$ ) conditions. Endothelial cell apoptosis was measured by counting TUNEL positive cells per 100 endothelial cells following the manufacturer's instructions (Promega, WI). Caspase-3 activity was measured using the caspase-3 kit (Sigma, MO).

2.3. Immunoprecipitation of Tie-2 and Blotting with SHP1 or Phospho-Tyrosine. MHMEC lysates were immunoprecipitated with anti-mouseTie-2 antibody followed by incubation with a $1: 1$ protein A: protein G-sepharose slurry. The immunoprecipitates were then subjected to SDSPAGE gels and transferred to nitrocellulose membranes. The membranes were immunoblotting anti-SHP- $1(1: 1000$, Santa Cruz, CA) or anti-phospho-tyrosine (4G10, 1:1000 Upstate Biotech, NY). The membranes were washed and incubated with a secondary antibody coupled to horseradish peroxidase.

2.4. SHP-1, Tie-2, Akt, and eNOS Expression. Fifty micrograms of total protein of myocardial tissue or MHMEC

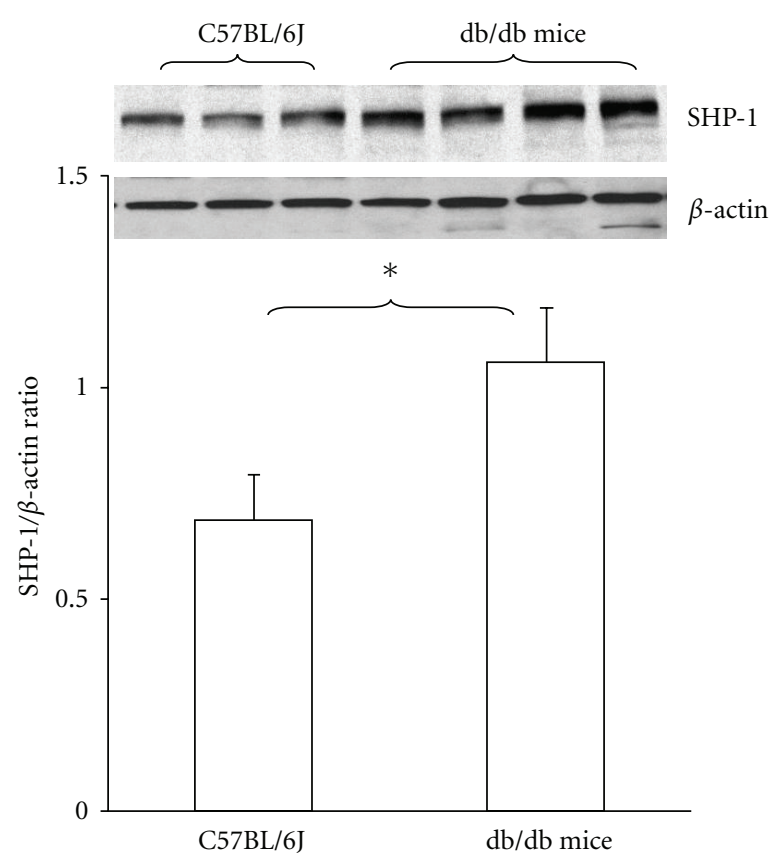

Figure 1: Expression of SHP-1 in the control C57BL/6J and db/db mouse hearts. Western blot showing SHP-1 expression in control $\mathrm{C} 57 \mathrm{BL} / 6 \mathrm{~J}$ and diabetic $\mathrm{db} / \mathrm{db}$ mouse hearts. Densitometric data shows that SHP-1 protein expression was significantly increased in diabetic $\mathrm{db} / \mathrm{db}$ mouse hearts in comparison with control C57BL/6J mice $\left(n=3-4\right.$ mice, $\left.{ }^{*} P<0.05\right)$.

lysates were separated using SDS-gel electrophoresis. The membranes were immunoblotted with SHP-1 $(1: 1000)$, eNOS and Tie-2 (1:1000, Cell Signaling Technology, MA) antibodies. For eNOS and Akt phosphorylation, the membranes were immunoblotted with rabbit anti-phospho-Akt and anti-phospho-eNOS $(1: 1000$, Cell Signaling, MA). $\beta$ Actin was used as a loading control $(1: 1000$, Cell Signaling Technology, MA) on the same nitrocellulose blots after stripping. The membranes were washed and incubated with a secondary antibody coupled to horseradish peroxidase, and densitometric analysis was carried out using image acquisition and analysis software (TINA 2.0).

2.5. SHP-1 siRNA Transfection. MHMEC (approx. 80\% confluent) was treated with SHP-1 siRNA (mouse, Santa Cruz, CA) for 24 hours to inhibition of SHP-1 expression according to the manufacturer's instructions. Knockdown of SHP-1 was confirmed by Western blot analysis of SHP-1 protein expression (data not shown).

2.6. Measurement of MHMEC Survival by MTT Assay. Cell survival was assayed using the MTT assay kit (Roche Diagnostic Corp., Indianapolis, IN).

2.7. Systemic Delivery of a PTP Inhibitor in Diabetic $d b / d b$ Mice. The C57BL/6J mice and $\mathrm{db} / \mathrm{db}$ mice were purchased from Jackson Laboratory (Bar Harbor, Maine). Sixteen male $\mathrm{db} / \mathrm{db}$ mice at 12 weeks of age were divided into two groups: [1] the PTP inhibitor treatment group: $\mathrm{db} / \mathrm{db}$ mice $(n=8)$ 


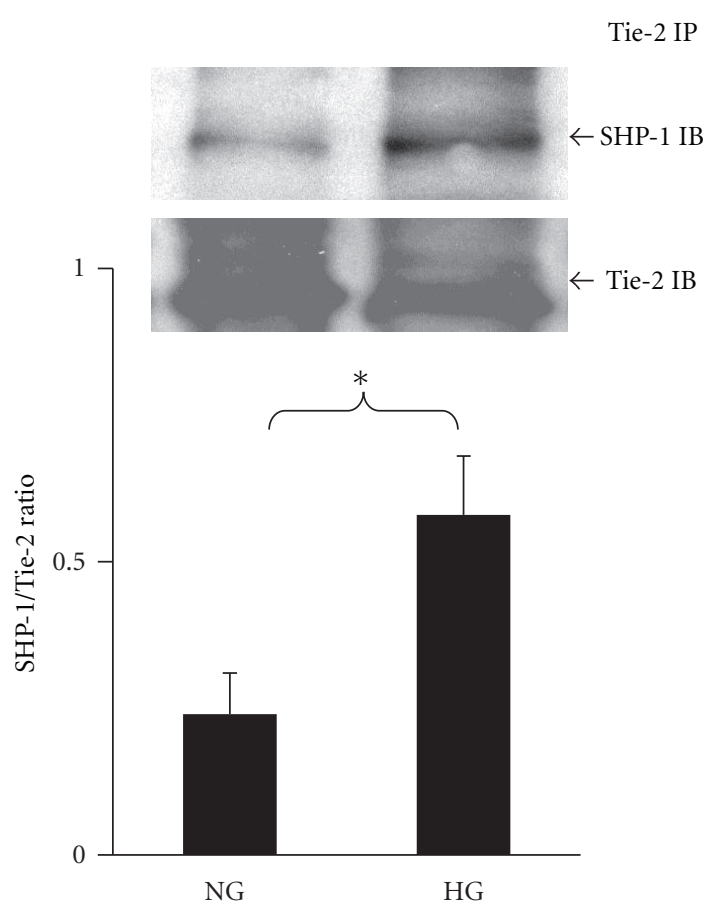

(a)

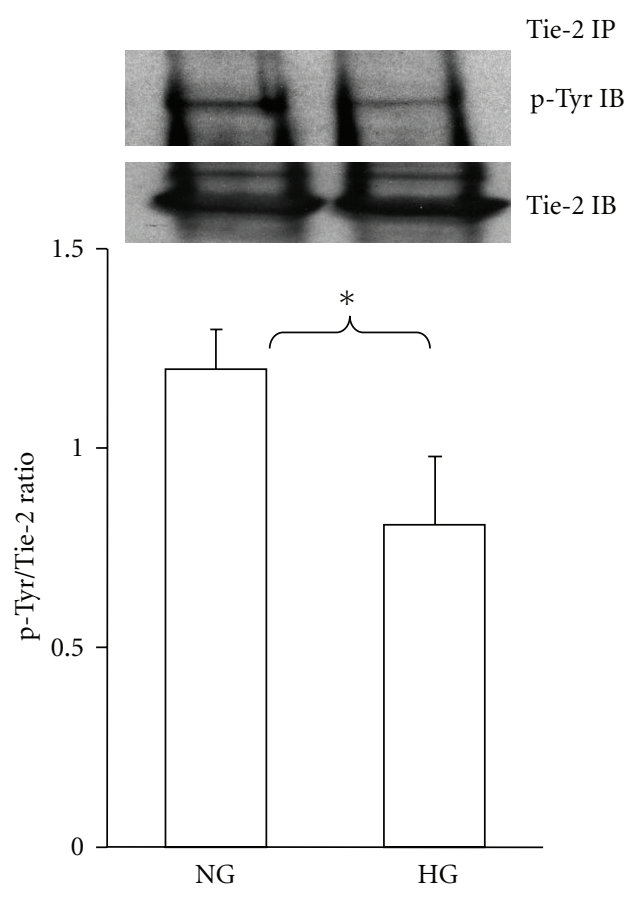

(b)

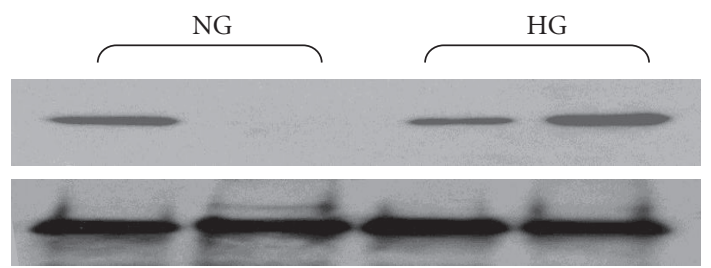

Tie-2 IP

SHP-1 IB

Tie-2 IB

Ang-1

(c)

FIGURE 2: Immunoprecipitation and Western blot analysis showing that SHP-1 associates with Tie-2 receptor and high glucose alters the SHP-1/Tie-2 association in MHMEC. (a) SHP-1 binds to the Tie-2 receptor under normal glucose (NG) conditions; exposure of MHMEC to high glucose (HG) leads to a significant increase in the SHP-1/Tie- 2 association $\left(n=4\right.$ cell lines, $\left.{ }^{*} P<0.05\right)$. (b) Exposure of MHMEC to high glucose (HG) conditions results in a significant reduction of Tie-2 phosphorylation $\left(n=3\right.$ cell lines, $\left.{ }^{*} P<0.05\right)$. (c) Under normal glucose (NG) conditions, stimulation of MHMEC with Ang-1 (250 ng/mL) for 15 minutes leads to a dissociation of SHP-1 from Tie-2. Under HG conditions, Ang-1 failed to induce SHP-1/Tie-2 dissociation. These results represent three different cell lines and experiments.

received oral bioavailable organovanadium compound, bis(maltolato)oxovanadium (IV) (BMOV, $0.2 \mathrm{~g} / \mathrm{L}$ ) in their drinking water for 2 weeks; [2] the $\mathrm{db} / \mathrm{db}$ control group received drinking water alone for 2 weeks. All procedures were in compliance with the Institute for Laboratory Animal Research Guide for the Care and Use of Laboratory Animals and were approved by the University of Mississippi Medical Center Institutional Animal Care and Use Committee.

2.8. In Vivo Myocardial Capillary Density Analysis. The experimental mouse hearts were harvested and immediately flash frozen. Tissue sections were incubated with fluoresceinlabeled antibodies against Griffonia Bandeiraea Simplicifolia Isolectin B4 (IB4, $1: 200$, Sigma Co) to label the endothelial cells, and myocardial capillary density was measured using image analysis software (Image J, NIH). The number of capillaries (IB4) was counted and expressed as capillary density per $\mathrm{mm}^{2}[9-11]$.

2.9. Ex Vivo Angiogenesis Assay. Mouse aortae were isolated and collected from C57BL/6J and $\mathrm{db} / \mathrm{db}$ mice, placed in the middle of organ culture dishes and overlaid with $300 \mu \mathrm{L}$ of ECM gel (Sigma Co, MO). After solidification, the ECM gel was covered with $10 \%$ FBS EGM in the presence or absence of recombinant human Ang-1 $(250 \mathrm{ng} / \mathrm{mL})$. Vessel outgrowth at day 5 was examined using a Nikon TE-300 microscope. The area of vessel outgrowth was quantified using image acquisition and analysis software (Image J, NIH) $[9,11,25,26]$. 


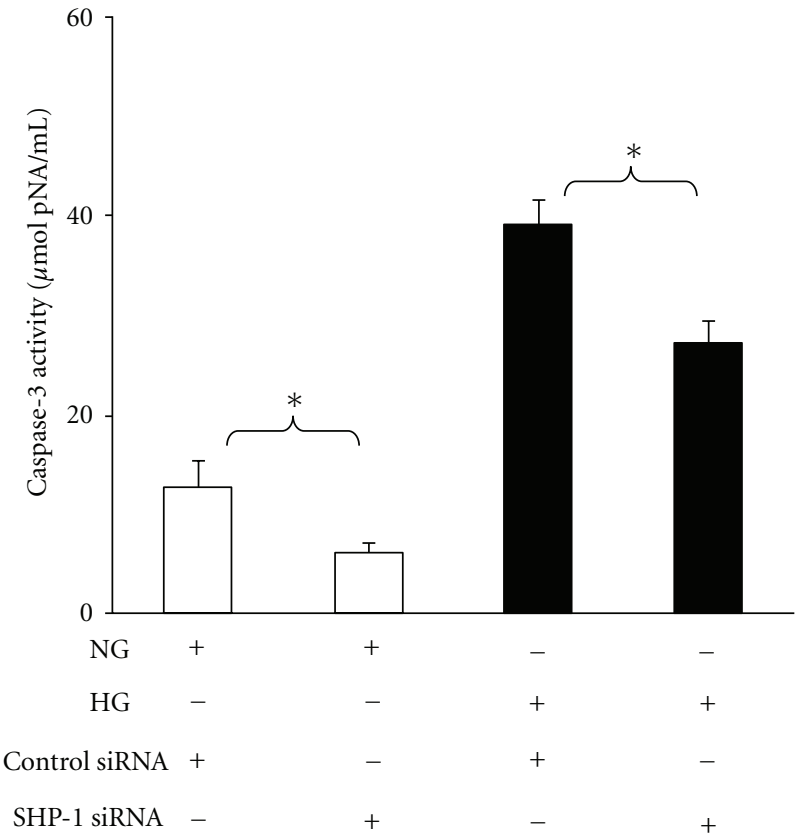

(a)

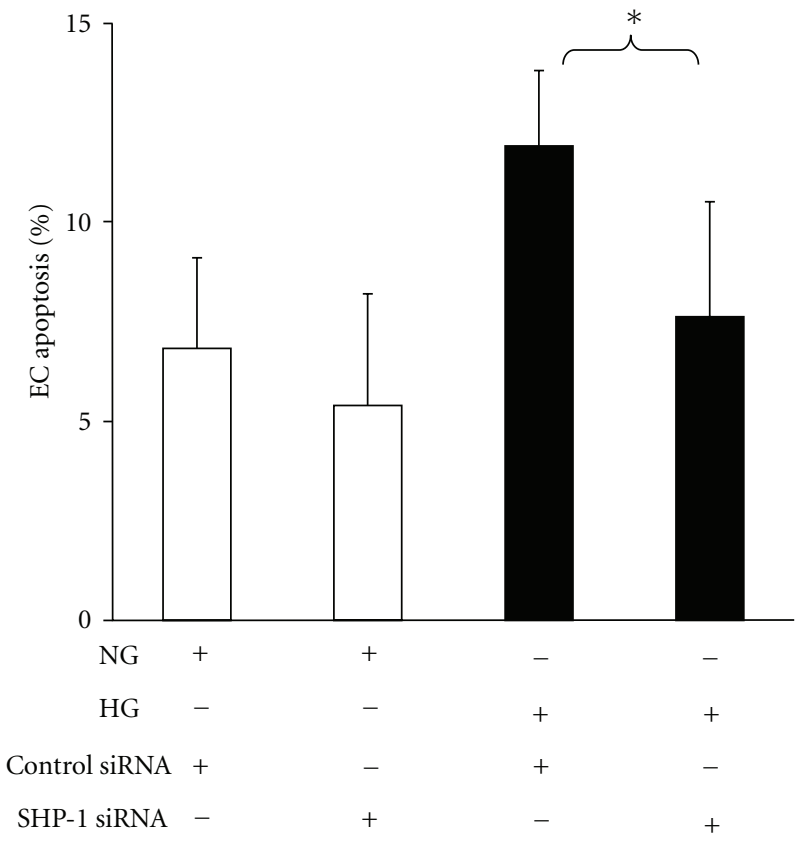

(b)

FIGURE 3: Knockdown of SHP-1 by siRNA blunts HG-induced cell apoptosis in MHMEC. (a) Caspase-3 ELISA analysis showing that transfection of MHMEC with SHP-1 siRNA significantly attenuated caspase-3 activation under NG or HG conditions $(n=3$ cell lines, $\left.{ }^{*} P<0.05\right)$. (b) TUNEL staining showing that MHMEC transfected with SHP-1 siRNA significantly inhibited high glucose-induced endothelial cell apoptosis $\left(n=3\right.$ cell lines, $\left.{ }^{*} P<0.05\right)$.

2.10. Statistical Analysis. All results were expressed as mean \pm SD. Statistical analysis was performed using unpaired student $t$-test. A $P$ value $<0.05$ denoted significance.

\section{Results}

3.1. SHP-1 Expression Is Upregulated in the Diabetic $d b / d b$ Mouse Hearts. Western blot analysis showed that SHP1 protein was expressed both in C57BL/6J mouse and diabetic $\mathrm{db} / \mathrm{db}$ mouse hearts. Intriguingly, the expression of SHP-1 protein was significantly increased in $\mathrm{db} / \mathrm{db}$ mouse hearts in comparison to C57BL/6J controls (Figure 1). The SHP-2 protein expression was unchanged in $\mathrm{db} / \mathrm{db}$ mouse hearts in comparison to C57BL/6J controls (data not shown).

3.2. HG Increases SHP-1/Tie-2 Association and Decreases Tie2 Tyrosine Phosphorylation in MHMEC. To examine whether SHP-1 binds to Tie-2, MHMEC lysates were immunoprecipitated with Tie-2 antibody and blotted with SHP-1 antibody. As shown in Figure 2(a), SHP-1 bond to Tie-2 and formed a Tie-2/SHP-1 complex. Exposure of MHMEC to HG $(30 \mathrm{mmol} / \mathrm{L})$ resulted in a significant increase in SHP-1/Tie-2 association. This was accompanied by a significant decrease in Tie-2 tyrosine phosphorylation (Figure 2(b)).

3.3. Ang-1 Induces SHP-1 Dissociation from Tie-2 and This Effect Is Ameliorated by $H G$ in MHMEC. To determine whether SHP-1 was involved in Ang-1-mediated Tie-2 activation, the effect of Ang-1 on Tie-2/SHP-1 association was examined. As shown in Figure 2(c), stimulation of MHMEC with Ang-1 $(250 \mathrm{ng} / \mathrm{mL})$ resulted in a dissociation of SHP-1 from Tie-2 receptor. Ang-1 failed to cause SHP-1dissociation from Tie-2 under HG conditions (Figure 2(c)).

3.4. SHP-1 siRNA Attenuates HG-Induced Caspase-3 Activation and Apoptosis in MHMEC. Next, the functional role of SHP-1 in high glucose-induced endothelial dysfunction was investigated. Treatment of MHMEC with SHP-1 siRNA significantly suppressed caspase-3 activity under normal glucose (NG) and HG conditions (Figure 3(a)). Further, treatment of MHMEC with SHP-1 siRNA significantly blunted HG-induced endothelial cell apoptosis (Figure 3(b)).

3.5. Inhibition of PTP Promotes Ang-1-Induced Angiogenic Signaling under HG Conditions. To further investigate the role of PTP in the Ang-1/Tie-2 signaling, the PTP inhibitor on Ang-1-induced Akt/eNOS phosphorylation was examined in MHMEC under HG conditions. MHMEC was pretreated with PTP inhibitor sodium orthovanadate $(\mathrm{OV}$, $5 \mu \mathrm{M})$ for 30 minutes, followed by Ang-1 $(250 \mathrm{ng} / \mathrm{mL})$ treatment for 15 minutes. Exposure of MHMEC to OV significantly enhanced Ang-1-induced Akt and eNOS phosphorylation under HG conditions. Pretreatment of MHMEC with OV alone had no significant effect on Akt and eNOS phosphorylation under HG conditions (Figures 4(b) and $4(\mathrm{~b}))$. 


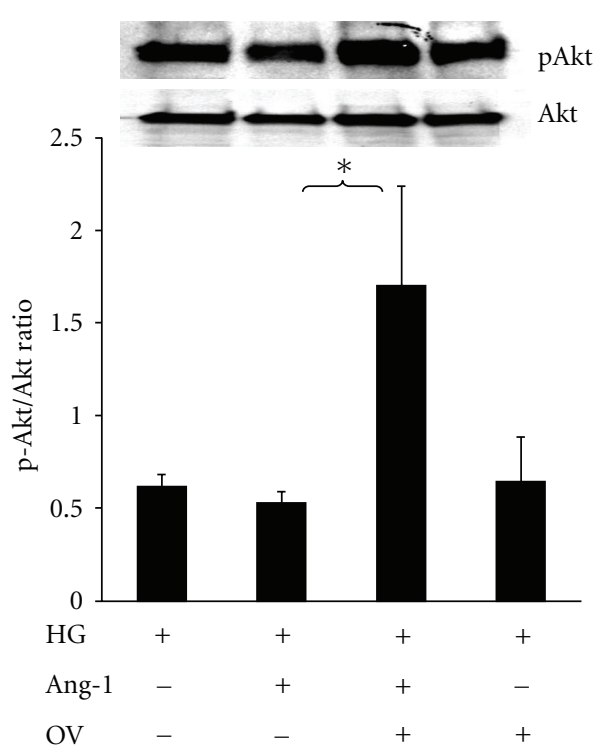

(a)

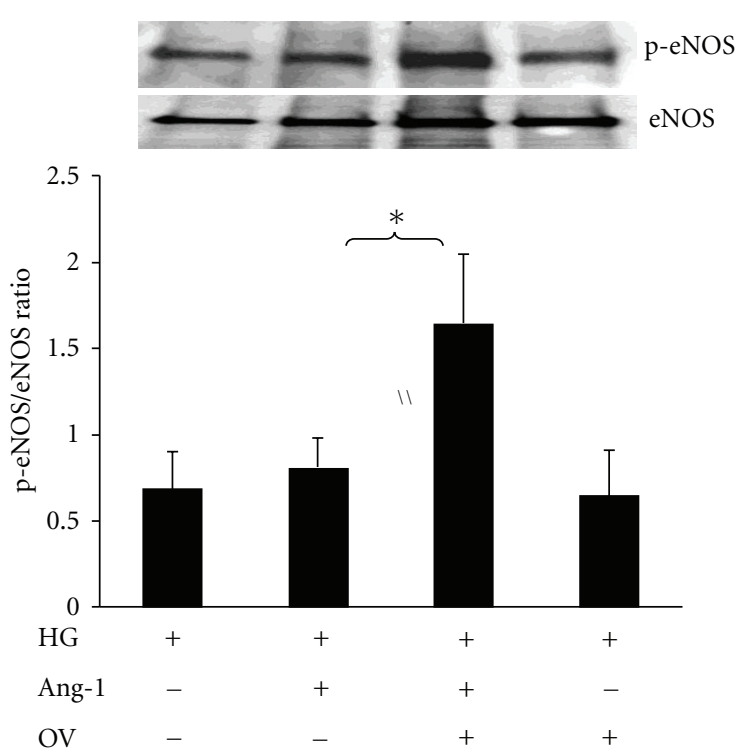

(b)

FIGURE 4: Inhibition of PTP enhances Ang-1-induced Akt and eNOS phosphorylation under HG conditions. (a) Western blot showing that pretreatment of MHMEC with the PTP inhibitor orthovanadate (OV, $5 \mu \mathrm{M})$ for 30 minutes leads to an increase in Akt phosphorylation in response to Ang-1 $(250 \mathrm{ng} / \mathrm{mL})$ stimulation under high glucose conditions $\left(n=3,{ }^{*} P<0.05\right)$. (b) Western blot showing that pretreatment of MHMEC with the PTP inhibitor OV for 30 minutes results in a significant increase in Ang-1-induced eNOS phosphorylation under high glucose conditions $(n=3, * P<0.05)$.

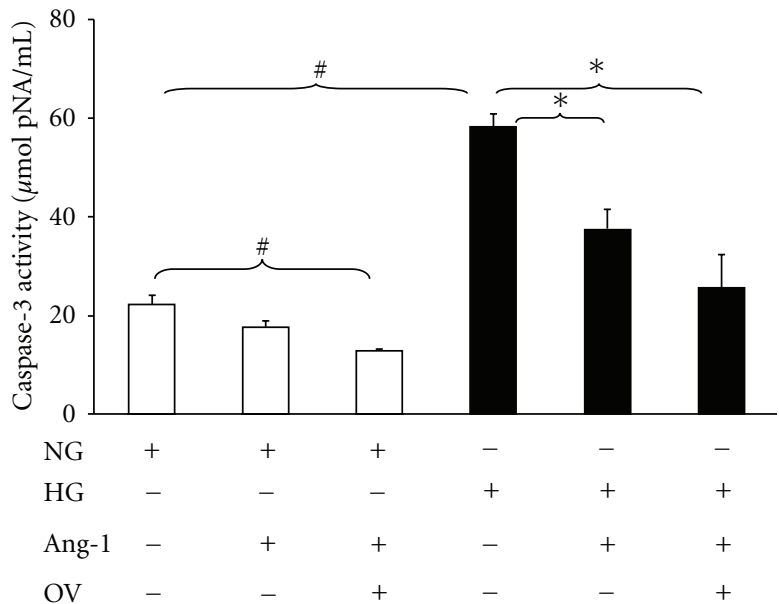

(a)

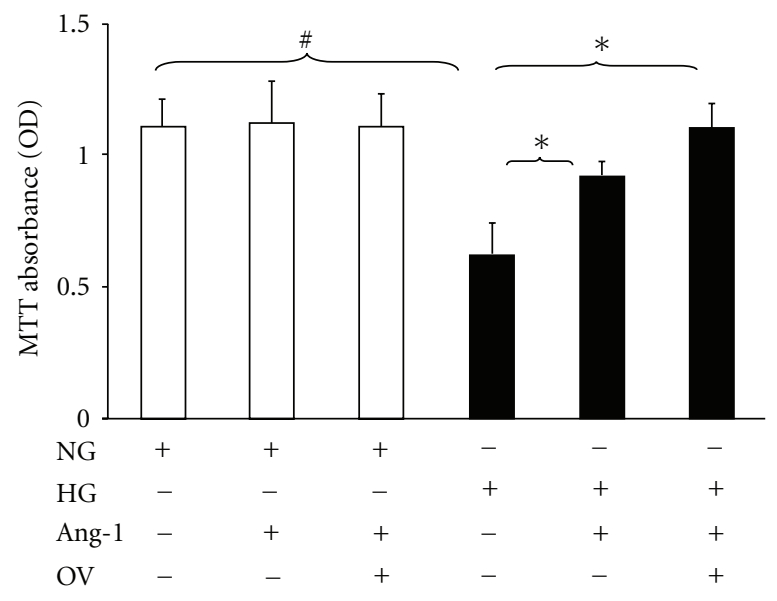

(b)

FIGURE 5: Inhibition of PTP promotes Ang-1-induced suppression of caspase-3 activation and increases cell survival under HG conditions. (a) Caspase-3 activity assay showing that exposure of MHMEC to Ang-1 significantly attenuates HG-induced caspase-3 activation; pretreatment of MHMEC with OV before the Ang-1 treatment further enhances the effect of Ang-1 on caspase-3 activity under HG conditions ( $n=3-5$ cell lines, ${ }^{*} P<0.05$ versus NG, ${ }^{*} P<0.05$ versus HG). (b) MTT assay for the cell survival showing that pretreatment with Ang-1 increases endothelial cell survival; pretreatment with OV before the Ang-1 treatment further enhances the effect of Ang-1 on endothelial cell survival under HG conditions ( $n=3-5$ cell lines, ${ }^{\#} P<0.05$ versus $N G,{ }^{*} P<0.05$ versus HG).

3.6. Inhibition of PTP Enhances Ang-1-Mediated Cell Survival in MHMEC. Treatment of MHMEC with Ang-1 $(250 \mathrm{ng} / \mathrm{mL})$ significantly attenuated caspase- 3 activity. The inhibitory effect of Ang-1 on caspase-3 activity was further enhanced in the presence of OV (Figure 5(a)). Ang-1 has a critical role in the regulation of endothelial cell survival. Treatment of MHMEC with Ang-1 (250 ng/mL) also increased cell survival under HG conditions. Similarly, 
C57BL/6 + Ang-1

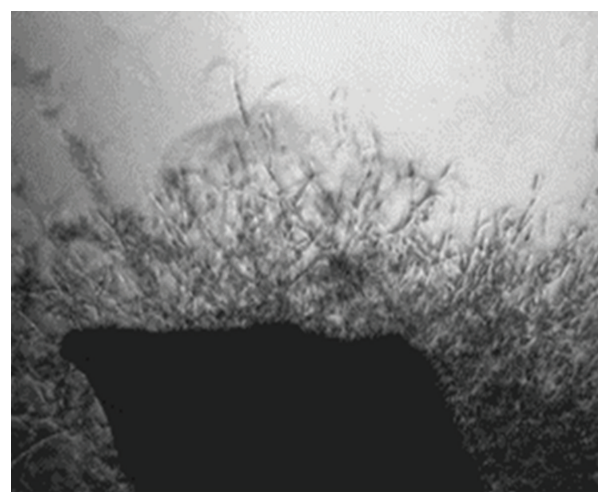

(a)

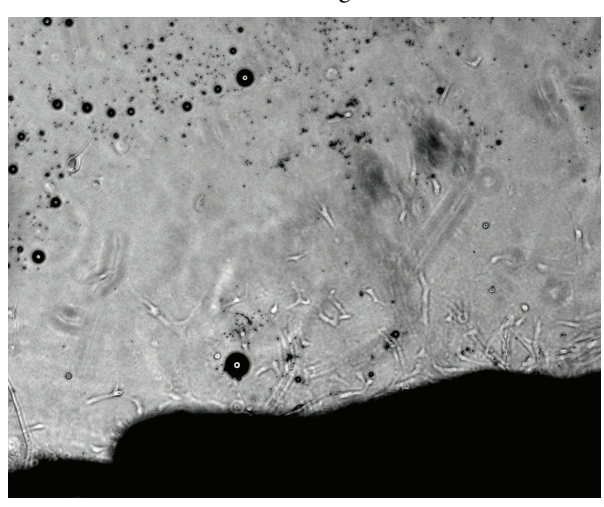

$\mathrm{db} / \mathrm{db}+$ Ang- 1

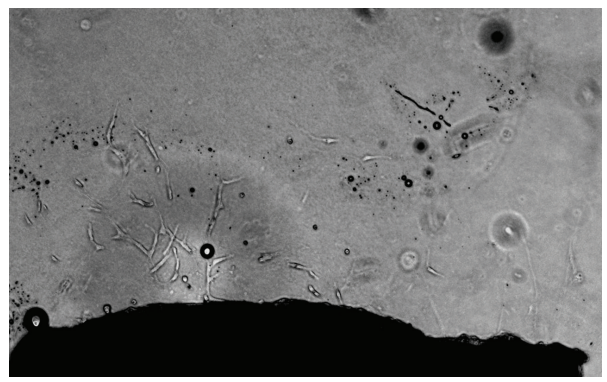

$\mathrm{db} / \mathrm{db}+$ Ang-1 + OV

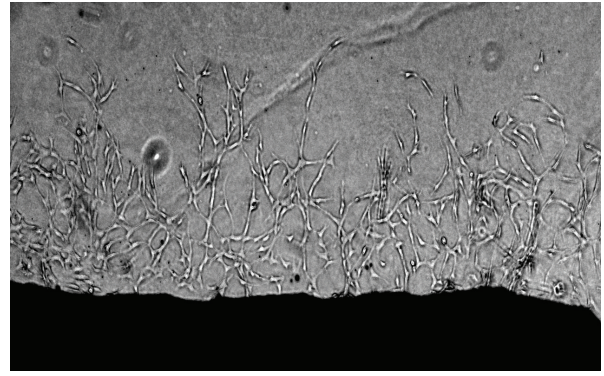

(b)

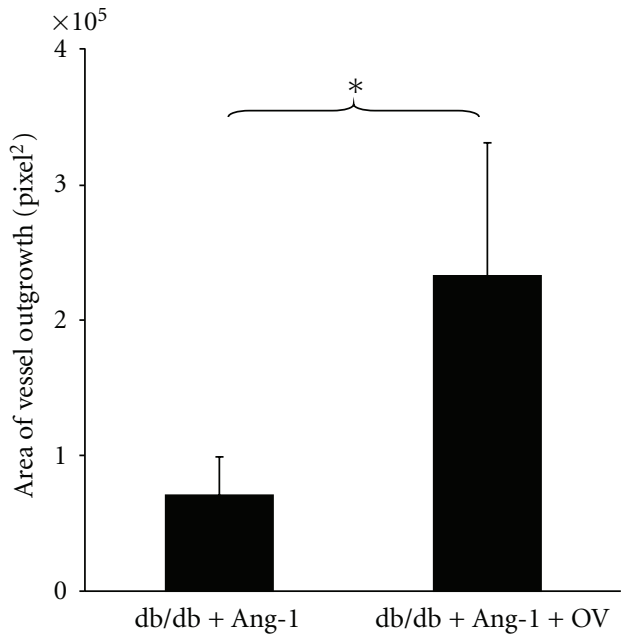

(c)

FIgURE 6: Treatment with PTP inhibitor restores the angiogenic response to Ang-1 in diabetic db/db mouse. (a) Representative images of Ang-1-induced aortic explants sprouting in C57BL/6J and diabetic db/db mice. Stimulation of C57BL/6J mouse aortic explants with Ang-1 (250 ng/mL) leads to robust vessel outgrowth. Ang-1-induced vessel outgrowth was blunted in the db/db mouse aortic explants. (b) Representative images of Ang-1-induced vessel outgrowth in the $\mathrm{db} / \mathrm{db}$ mouse aortic explants in the presence and absence of PTP inhibitor OV. (c) Quantitative areas of vessel outgrowth in $\mathrm{db} / \mathrm{db}$ mouse aortic explants showing Ang-1-induced vessel outgrowth were significantly increased in the presence of PTP inhibitor OV $\left(n=4-8\right.$ mice, $\left.{ }^{*} P<0.05\right)$. 


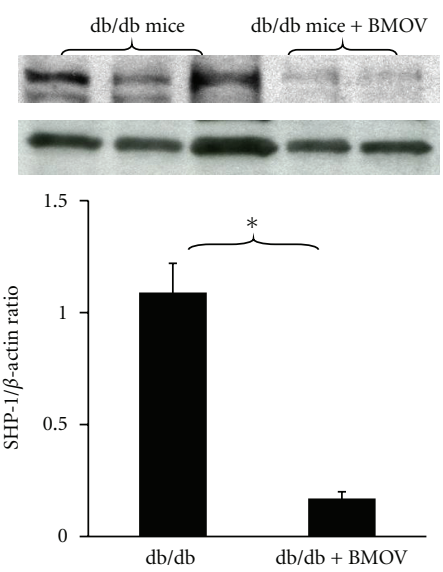

(a)
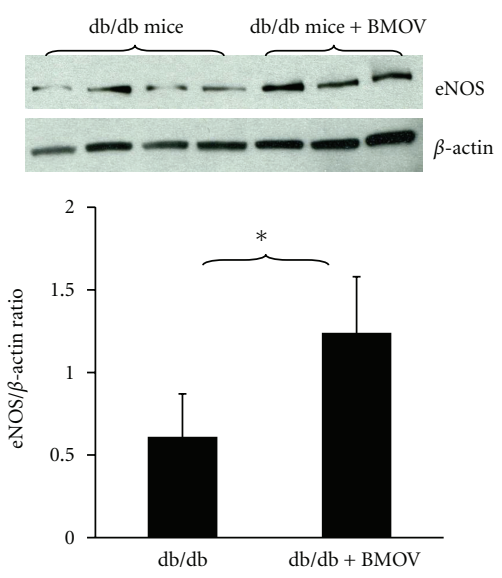

(b)
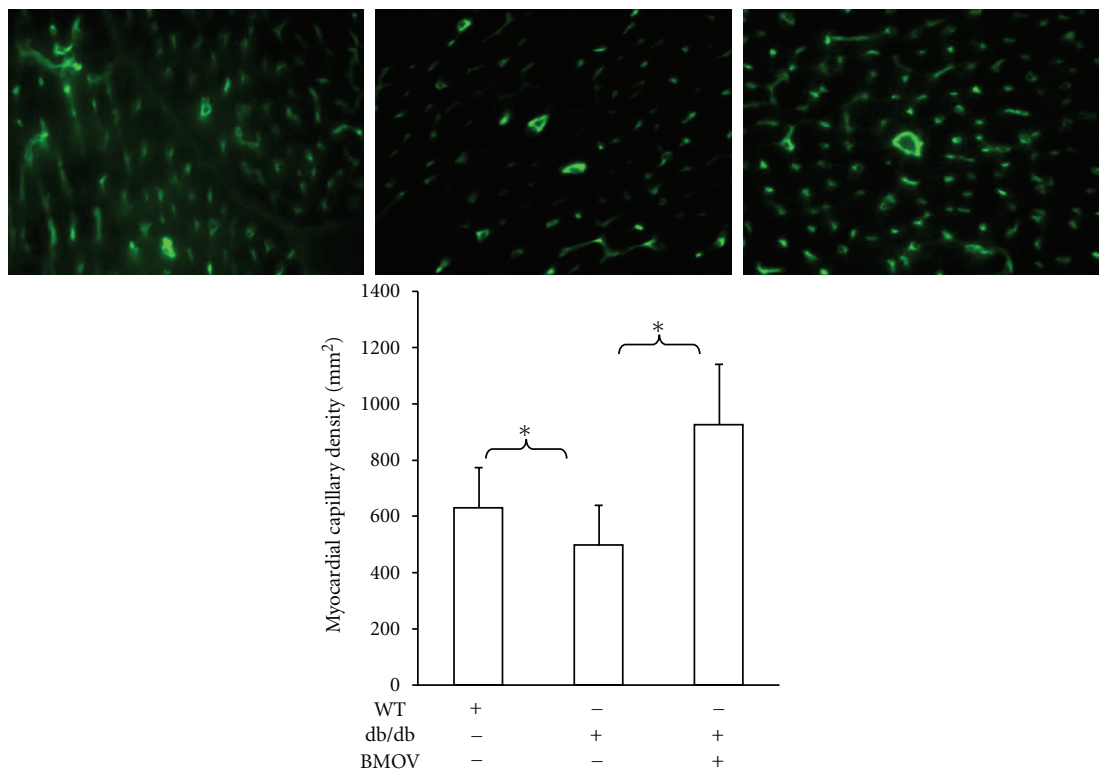

(c)

$\mathrm{db} / \mathrm{db}$

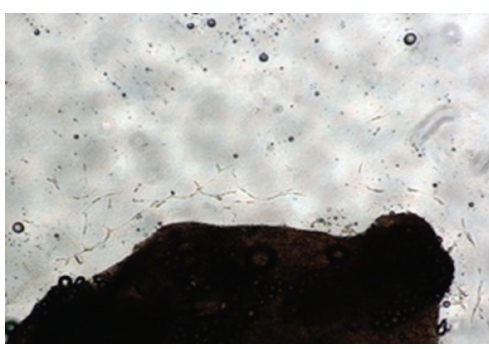

$\mathrm{db} / \mathrm{db} / \mathrm{BMOV}$

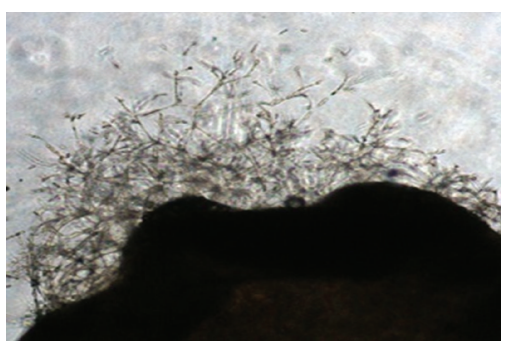

(d)

FIGURE 7: Systemic treatment with the PTP inhibitor attenuates SHP-1 expression and increases eNOS and myocardial capillary density in $\mathrm{db} / \mathrm{db}$ mice. (a) Western blot analysis showing that treatment with BMOV for 2 weeks leads to a significant suppression of SHP-1 expression in the $\mathrm{db} / \mathrm{db}$ mice compared to $\mathrm{db} / \mathrm{db}$ control without BMOV $\left(n=3\right.$ mice, $\left.{ }^{*} P<0.05\right)$. (b) Western blot analysis showing that treatment with BMOV for 2 weeks results in a significant increase in eNOS expression in the $\mathrm{db} / \mathrm{db}$ mice compared to $\mathrm{db} / \mathrm{db}$ control $(n=3-4 \mathrm{mice}$, $\left.{ }^{*} P<0.05\right)$. (c) Myocardial capillary density was significantly increased in the BMOV-treated $\mathrm{db} / \mathrm{db}$ mice compared to $\mathrm{db} / \mathrm{db}$ control with BMOV $(n=8, * P<0.05)$. (d) Quantitation of areas of vessel outgrowth showing Ang-1-induced vessel explants sprouting is significantly improved in the BMOV-treated $\mathrm{db} / \mathrm{db}$ mice compared to $\mathrm{db} / \mathrm{db}$ control group $\left(n=4-8\right.$ mice, $\left.{ }^{*} P<0.05\right)$. 


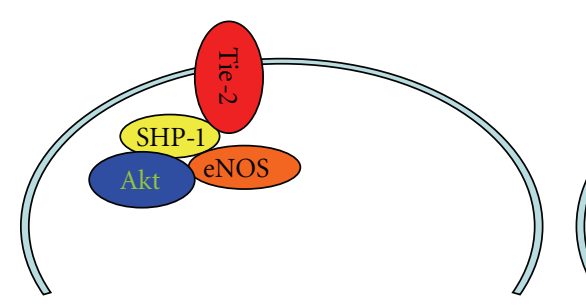

(a)

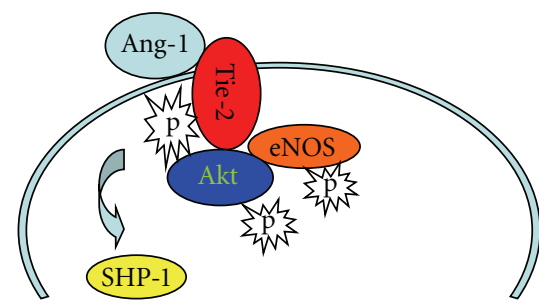

(b)

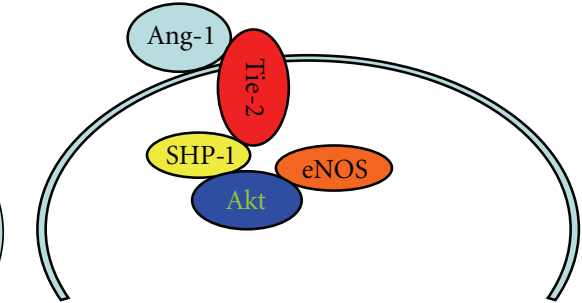

(c)

FIGURE 8: Illustrating our working hypothesis for the SHP-1-induced disruption of Ang-1/Tie-2 signaling under HG conditions and in diabetes. (a) In a resting state, SHP-1 maintains Tie-2 inactivation. (b) Stimulation with Ang-1 causes a dissociation of SHP-1 and Tie-2, thus leading to Tie-2 tyrosine phosphorylation, and its downstream signaling Akt and eNOS activation. (c) Stimulation with Ang-1 fails to lead to the dissociation of SHP-1 and Tie-2, thus resulting in a disruption of Ang-1/Tie-2 signaling under hyperglycemic conditions and in diabetes. NG: normal glucose; HG: high glucose; SHP-1: protein tyrosine phosphatase-1.

Ang-1-induced cell survival was greatly enhanced in the presence of OV under HG conditions. Surprisingly, OV alone had little effect on cell survival (Figure 5(b)).

\subsection{Inhibition of PTP Augments Ang-1-Induced Vessel Out-} growth in $d b / d b$ Mouse. As shown in Figure 6(a), exposure of C57BL/6J aortic explants to Ang-1 $(250 \mathrm{ng} / \mathrm{mL})$ resulted in a robust angiogenic response. In contrast, the Ang-1induced vessel outgrowth was greatly diminished in $\mathrm{db} / \mathrm{db}$ mouse vessel explants compared with C57BL/6J mouse. In the presence of OV, Ang-1-induced vessel outgrowth was significantly augmented compared to the $\mathrm{db} / \mathrm{db}$ control group (Figures 6(b) and 6(c)).

3.8. Inhibition of PTP Increases eNOS Expression and Capillary Density in $d b / d b$ Mouse Hearts. To examine whether inhibition of PTP augments myocardial angiogenesis in diabetic hearts, an orally bioavailable PTP inhibitor bis(maltolato)oxovanadium (IV) (BMOV) was given to the experimental $\mathrm{db} / \mathrm{db}$ mice. Treatment of $\mathrm{db} / \mathrm{db}$ mice with BMOV for 2 weeks resulted in a significant decrease in SHP-1 expression (Figure 7(a)). This was accompanied by a significant increase in eNOS expression in $\mathrm{db} / \mathrm{db}$ mouse hearts (Figure 7(b)). Immunohistochemical studies revealed that myocardial capillary density was significantly decreased in $\mathrm{db} / \mathrm{db}$ mouse hearts when compared to C57BL/6J controls. Myocardial capillary density was significantly increased in the BMOV-treated $\mathrm{db} / \mathrm{db}$ mice (Figure $7(\mathrm{c})$ ). Our ex vivo angiogenesis studies also showed that Ang-1-induced vessel outgrowth was significantly improved in the BMOVtreated $\mathrm{db} / \mathrm{db}$ mice in comparison with $\mathrm{db} / \mathrm{db}$ control group (Figure 7(d)).

\section{Discussion}

The current study demonstrates that SHP-1 binds to the Tie-2 to form a SHP-1/Tie-2 complex and that the Ang1 , an agonist of Tie-2, causes SHP-1 dissociation from Tie-2. This finding implicates a potential role of SHP-1 in Ang-1-induced Tie-2 phosphorylation. Intriguingly, high glucose increases formation of the SHP-1/Tie-2 complex and this is accompanied by Tie-2 dephosphorylation. Ang1 failed to cause SHP-1 dissociation from Tie-2 under HG conditions. Suppression of SHP-1 expression significantly attenuated endothelial apoptosis and improved diabetesassociated impairment of angiogenesis. These data strongly suggest a critical role for SHP-1 and the SHP-1/Tie-2 association in diabetes-associated impairment of angiogenesis.

The Src-homology-domain-2- (SH2-) containing tyrosine phosphatases (SHP-1 and SHP-2) have been shown to interact with multiple growth factor receptors including Tie$2[27,28]$. SHP-2 is primarily associated with enhanced cell growth, whereas SHP-1 has been shown to play a negative regulatory role in endothelial cell proliferation $[18,28-30]$. SHP-1 suppresses VEGF and EGF-induced endothelial proliferation, whereas knockdown of SHP-1 augments VEGFand FGF-2-induced angiogenic responses [20, 31]. SHP1 also showed to negatively modulate glucose homeostasis via de-phosphorylation of insulin RTK signaling [32]. Our study demonstrated that SHP-1 expression was significantly increased whereas SHP-2 expression remained unchanged in diabetic $\mathrm{db} / \mathrm{db}$ mouse hearts. Our present study also demonstrated that SHP-1 works as a novel client protein for Tie-2, and stimulation with Ang-1 led to SHP-1 dissociation from Tie-2, implicating a potential interaction between SHP1 and Ang-1-induced Tie-2 phosphorylation. This notion was further validated by our finding that exposure of MHMEC to HG increased SHP-1/Tie-2 association but decreased Tie-2 phosphorylation. This was consistent with our previous studies that Ang-1-induced Tie-2 phosphorylation was damped under HG conditions [33]. Taken together, the present study reveals a potential novel mechanism for the disruption of Ang-1/Tie-2 signaling by SHP-1 in diabetes. We speculate that protein tyrosine phosphatases, including SHP-1, maintain Tie-2 inactivation by de-phosphorylation, whereas stimulation with Ang-1 leads to dissociation of SHP-1 from Tie-2 and results in Tie-2 phosphorylation and its downstream signaling Akt and eNOS activation. Under hyperglycemic conditions and in diabetes, stimulation with Ang-1 fails to cause the dissociation of SHP-1 from Tie-2, resulting in disruption of Ang-1/Tie-2 signaling (Figure 8).

Our data also demonstrated that knockdown of SHP1 by siRNA significantly prevented HG-induced caspase- 3 
activation and endothelial apoptosis. Our study further demonstrates that inhibition of PTP augmented Ang-1induced cell survival under HG conditions and restored angiogenic responses in diabetic vessel explants. Inhibition of PTP has been shown to enhance angiogenic signaling and promote VEGF-induced angiogenesis [34]. Inhibition of PTP also promoted collateral blood vessel formation and increased blood flow in a rat model of hind-limb ischemia [14, 15]. Inhibition of PTP has been shown to attenuate endothelial dysfunction via upregulation of eNOS in the mouse model of chronic heart failure [35] and treatment with the nonselective PTP inhibitors such as vanadate and BMOV-enhanced insulin receptor activation and restored insulin signaling in diabetic rats [36-38]. The protective effect of PTP inhibitors on endothelial cell dysfunction was mediated by the enhancement of Akt/eNOS phosphorylation in diabetic rats [36]. Consistent with these findings, our data showed that pretreatment of MHMEC with a PTP inhibitor enhanced Ang-1-induced Akt/eNOS phosphorylation. Our present study also demonstrated that systemic treatment of diabetic $\mathrm{db} / \mathrm{db}$ mouse with the PTP inhibitor BMOV significantly suppressed SHP-1 expression and increased eNOS expression. This was accompanied by increase in myocardial capillary density. Our study provides new evidence that diabetes may impede angiogenesis by a mechanism involving upregulation of PTP activity which negatively regulates angiogenesis by inhibition of angiogenic growth factor phosphorylation such as Ang-1/Tie-2 system.

4.1. Limitation of This Study. Other PTPs, including PTP1B, SHP-2, PTP- $\varepsilon$, VE-PTP, CD148, may also play key roles in the regulation of myocardial angiogenesis in diabetes. Further elucidation of the intracellular mechanisms of PTP with, such as, PTPB1 on diabetes-associated impairment of angiogenic signaling and angiogenesis is needed. We acknowledge that it is technically impossible to examine all PTPs enzymes in a similar manner since specific inhibitors are lacking for each individual isoform of the PTPs. We also acknowledge the potential integrated effects of SHP-1 and PKC beta signaling. Identification of all the mechanisms involved will require additional experiments to evaluate the roles of PTPs and PKC signaling pathways in diabetesassociated impairment of angiogenesis.

In summary, our present study demonstrates that hyperglycemia and diabetes impair angiogenesis by a mechanism involving upregulation of SHP-1 and SHP-1/Tie-2 association. Our study also shows that pharmacological inhibition of PTP or genetic deletion of SHP-1 enhances Ang-1/Tie2 signaling and improves angiogenesis in diabetes. Our data implicate that restoration of Ang-1/Tie-2 signaling by PTP inhibitors should be considered as a new therapeutic strategy for the treatment or prevention of diabetic impaired angiogenesis.

\section{Acknowledgment}

This work was supported by grant from HL102042 to J. X. Chen.

\section{References}

[1] N. W. Gale and G. D. Yancopoulos, "Growth factors acting via endothelial cell-specific receptor tyrosine kinases: VEGFs, angiopoietins, and ephrins in vascular development," Genes and Development, vol. 13, no. 9, pp. 1055-1066, 1999.

[2] J. M. van Golde, M. S. Ruiter, N. C. Schaper et al., "Impaired collateral recruitment and outward remodeling in experimental diabetes," Diabetes, vol. 57, no. 10, pp. 2818-2823, 2008.

[3] M. S. Ruiter, J. M. van Golde, N. C. Schaper, C. D. Stehouwer, and M. S. Huijberts, "Diabetes impairs arteriogenesis in the peripheral circulation: review of molecular mechanisms," Clinical Science, vol. 119, no. 6, pp. 225-238, 2010.

[4] A. Abaci, A. Oguzhan, S. Kahraman et al., "Effect of diabetes mellitus on formation of coronary collateral vessels," Circulation, vol. 99, no. 17, pp. 2239-2242, 1999.

[5] J. Waltenberger, "Impaired collateral vessel development in diabetes: potential cellular mechanisms and therapeutic implications," Cardiovascular Research, vol. 49, no. 3, pp. 554-560, 2001.

[6] J. Waltenberger, J. Lange, and A. Kranz, "Vascular endothelial growth factor-A-induced chemotaxis of monocytes is attenuated in patients with diabetes mellitus: a potential predictor for the individual capacity to develop collaterals," Circulation, vol. 102, no. 2, pp. 185-190, 2000.

[7] V. Tchaikovski, S. Olieslagers, F. D. Böhmer, and J. Waltenberger, "Diabetes mellitus activates signal transduction pathways resulting in vascular endothelial growth factor resistance of human monocytes," Circulation, vol. 120, no. 2, pp. 150-159, 2009.

[8] F. C. Sasso, D. Torella, O. Carbonara et al., "Increased vascular endothelial growth factor expression but impaired vascular endothelial growth factor receptor signaling in the myocardium of type 2 diabetic patients with chronic coronary heart disease," Journal of the American College of Cardiology, vol. 46, no. 5, pp. 827-834, 2005.

[9] J. X. Chen and A. Stinnett, "Disruption of Ang-1/Tie-2 signaling contributes to the impaired myocardial vascular maturation and angiogenesis in type II diabetic mice," Arteriosclerosis, Thrombosis, and Vascular Biology, vol. 28, no. 9, pp. 1606-1613, 2008.

[10] Q. H. Tuo, H. Zeng, A. Stinnett et al., "Critical role of angiopoietins/Tie-2 in hyperglycemic exacerbation of myocardial infarction and impaired angiogenesis," American Journal of Physiology, vol. 294, no. 6, pp. H2547-H2557, 2008.

[11] J. X. Chen and A. Stinnett, "Ang-1 gene therapy inhibits hypoxia-inducible Factor- $1 \alpha$ (HIF- $1 \alpha$ )-prolyl-4-hydroxylase2, stabilizes HIF- $1 \alpha$ expression, and normalizes immature vasculature in db/db mice," Diabetes, vol. 57, no. 12, pp. 33353343, 2008.

[12] S. Dadke, A. Kusari, and J. Kusari, "Phosphorylation and activation of protein tyrosine phosphatase (PTP) 1B by insulin receptor," Molecular and Cellular Biochemistry, vol. 221, no. 12, pp. 147-154, 2001.

[13] M. Elchebly, P. Payette, E. Michaliszyn et al., "Increased insulin sensitivity and obesity resistance in mice lacking the protein tyrosine phosphatase-1B gene," Science, vol. 283, no. 5407, pp. 1544-1548, 1999.

[14] M. Sugano, K. Tsuchida, and N. Makino, "A protein tyrosine phosphatase inhibitor accelerates angiogenesis in a rat model of hindlimb ischemia," Journal of Cardiovascular Pharmacology, vol. 44, no. 4, pp. 460-465, 2004.

[15] S. Soeda, T. Shimada, S. Koyanagi et al., "An attempt to promote neo-vascularization by employing a newly synthesized 
inhibitor of protein tyrosine phosphatase," FEBS Letters, vol. 524, no. 1-3, pp. 54-58, 2002.

[16] T. Yi, J. L. Cleveland, and J. N. Ihle, "Protein tyrosine phosphatase containing SH2 domains: characterization, preferential expression in hematopoietic cells, and localization to human chromosome 12p12-p13," Molecular and Cellular Biology, vol. 12, no. 2, pp. 836-846, 1992.

[17] B. G. Neel, H. Gu, and L. Pao, "The 'Shp'ing news: SH2 domain-containing tyrosine phosphatases in cell signaling," Trends in Biochemical Sciences, vol. 28, no. 6, pp. 284-293, 2003.

[18] H. Nakagami, T. X. Cui, M. Iwai et al., "Tumor necrosis factor- $\alpha$ inhibits growth factor-mediated cell proliferation through SHP-1 activation in endothelial cells," Arteriosclerosis, Thrombosis, and Vascular Biology, vol. 22, no. 2, pp. 238-242, 2002.

[19] Z. Z. Chong and K. Maiese, "The Src homology 2 domain tyrosine phosphatases SHP-1 and SHP-2: diversified control of cell growth, inflammation, and injury," Histology and Histopathology, vol. 22, no. 11, pp. 1251-1267, 2007.

[20] D. W. Seo, H. Li, L. Guedez et al., "TIMP-2 mediated inhibition of angiogenesis: an MMP-independent mechanism," Cell, vol. 114, no. 2, pp. 171-180, 2003.

[21] N. Wang, Z. Li, R. Ding et al., "Antagonism or synergism: role of tyrosine phosphatases SHP-1 and SHP-2 in growth factor signaling," The Journal of Biological Chemistry, vol. 281, no. 31, pp. 21878-21883, 2006.

[22] Z. U. A. Mollah, S. Pai, C. Moore et al., "Abnormal NF- $\kappa$ B function characterizes human type 1 diabetes dendritic cells and monocytes," The Journal of Immunology, vol. 180, no. 5, pp. 3166-3175, 2008.

[23] A. Östman and F. D. Böhmer, "Regulation of receptor tyrosine kinase signaling by protein tyrosine phosphatases," Trends in Cell Biology, vol. 11, no. 6, pp. 258-266, 2001.

[24] J. X. Chen, M. L. Lawrence, G. Cunningham, B. W. Christman, and B. Meyrick, "HSP90 and Akt modulate Ang-1-induced angiogenesis via NO in coronary artery endothelium," Journal of Applied Physiology, vol. 96, no. 2, pp. 612-620, 2004.

[25] J. X. Chen, H. Zeng, M. L. Lawrence, T. S. Blackwell, and B. Meyrick, "Angiopoietin-1-induced angiogenesis is modulated by endothelial NADPH oxidase," American Journal of Physiology, vol. 291, no. 4, pp. H1563-H1572, 2006.

[26] J. X. Chen, H. Zeng, Q. H. Tuo, H. Yu, B. Meyrick, and J. L. Aschner, "NADPH oxidase modulates myocardial Akt, ERK1/2 activation, and angiogenesis after hypoxiareoxygenation," American Journal of Physiology, vol. 292, no. 4, pp. H1664-H1674, 2007.

[27] L. K. Tai, Q. Zheng, S. Pan, Z. G. Jin, and B. C. Berk, "Flow activates ERK1/2 and endothelial nitric oxide synthase via a pathway involving PECAM1, SHP2, and Tie2," The Journal of Biological Chemistry, vol. 280, no. 33, pp. 29620-29624, 2005.

[28] J. Cai, W. G. Jiang, A. Ahmed, and M. Boulton, "Vascular endothelial growth factor-induced endothelial cell proliferation is regulated by interaction between VEGFR-2, SH-PTP1 and eNOS," Microvascular Research, vol. 71, no. 1, pp. 20-31, 2006.

[29] D. Q. Guo, L. W. Wu, J. D. Dunbar et al., "Tumor necrosis factor employs a protein-tyrosine phosphatase to inhibit activation of KDR and vascular endothelial cell growth factor-induced endothelial cell proliferation," The Journal of Biological Chemistry, vol. 275, no. 15, pp. 11216-11221, 2000.

[30] J. Kroll and J. Waltenberger, "The vascular endothelial growth factor receptor KDR activates multiple signal transduction pathways in porcine aortic endothelial cells," The Journal of Biological Chemistry, vol. 272, no. 51, pp. 32521-32527, 1997.

[31] D. W. Seo, H. Li, C. K. Qu et al., "Shp-1 mediates the antiproliferative activity of tissue inhibitor of metalloproteinase2 in human microvascular endothelial cell," The Journal of Biological Chemistry, vol. 281, no. 6, pp. 3711-3721, 2006.

[32] M. J. Dubois, S. Bergeron, H. J. Kim et al., "The SHP-1 protein tyrosine phosphatase negatively modulates glucose homeostasis," Nature Medicine, vol. 12, no. 5, pp. 549-556, 2006.

[33] Q. H. Tuo, G. Z. Xiong, H. Zeng et al., "Angiopoietin1 protects myocardial endothelial cell function blunted by angiopoietin-2 and high glucose condition," Acta Pharmacologica Sinica, vol. 32, no. 1, pp. 45-51, 2011.

[34] A. N. Carr, M. G. Davis, E. Eby-Wilkens et al., "Tyrosine phosphatase inhibition augments collateral blood flow in a rat model of peripheral vascular disease," American Journal of Physiology, vol. 287, no. 1, pp. H268-H276, 2004.

[35] M. Vercauteren, E. Remy, C. Devaux et al., "Improvement of peripheral endothelial dysfunction by protein tyrosine phosphatase inhibitors in heart failure," Circulation, vol. 114, no. 23, pp. 2498-2507, 2006.

[36] D. I. Shah and M. Singh, "Inhibition of protein tyrosin phosphatase improves vascular endothelial dysfunction," Vascular Pharmacology, vol. 44, no. 3, pp. 177-182, 2006.

[37] C. L. Winter, J. S. Lange, M. G. Davis et al., "A nonspecific phosphotyrosine phosphatase inhibitor, bis(maltolato) oxovanadium(IV), improves glucose tolerance and prevents diabetes in Zucker diabetic fatty rats," Experimental Biology and Medicine, vol. 230, no. 3, pp. 207-216, 2005.

[38] J. Meyerovitch, P. Rothenberg, Y. Shechter, S. Bonner-Weir, and C. R. Kahn, "Vanadate normalizes hyperglycemia in two mouse models of non-insulin-dependent diabetes mellitus," Journal of Clinical Investigation, vol. 87, no. 4, pp. 1286-1294, 1991. 


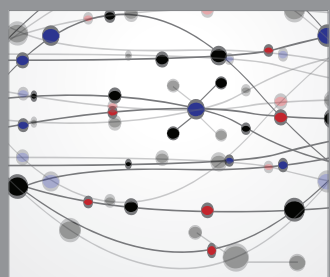

The Scientific World Journal
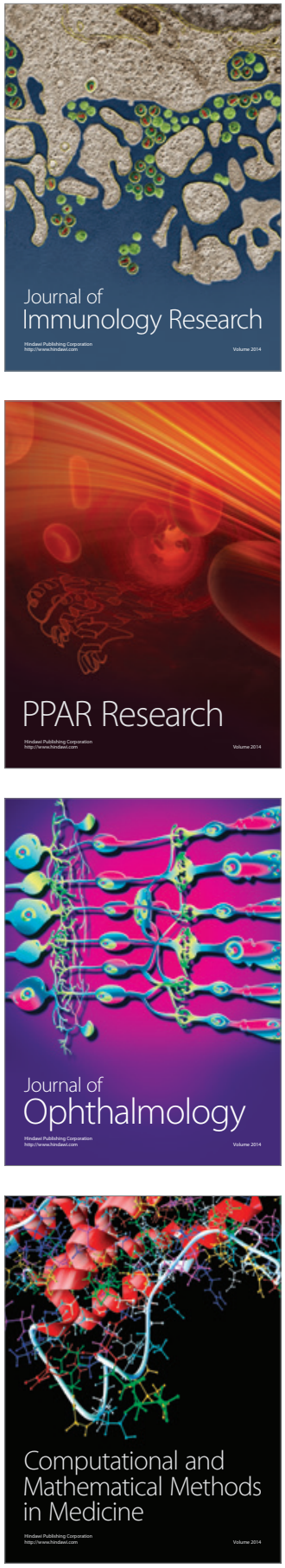

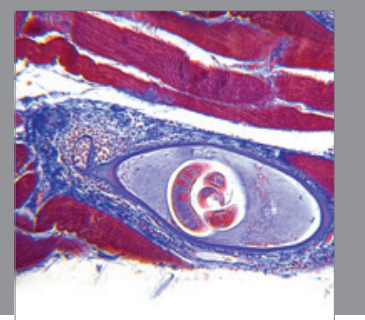

Gastroenterology

Research and Practice
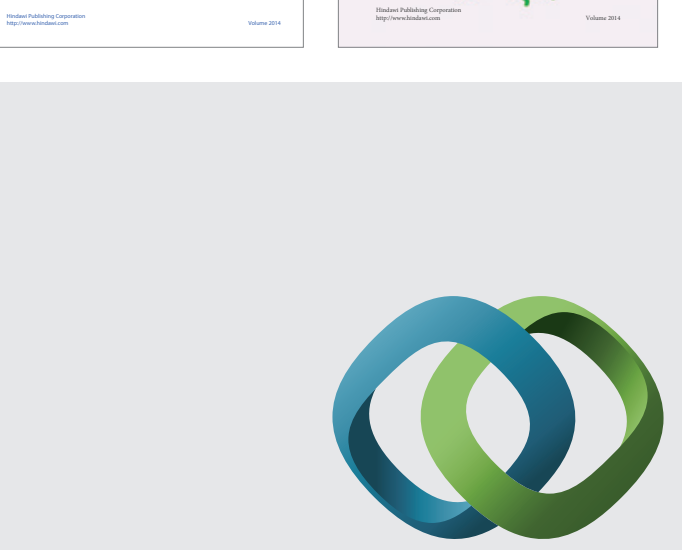

\section{Hindawi}

Submit your manuscripts at

http://www.hindawi.com
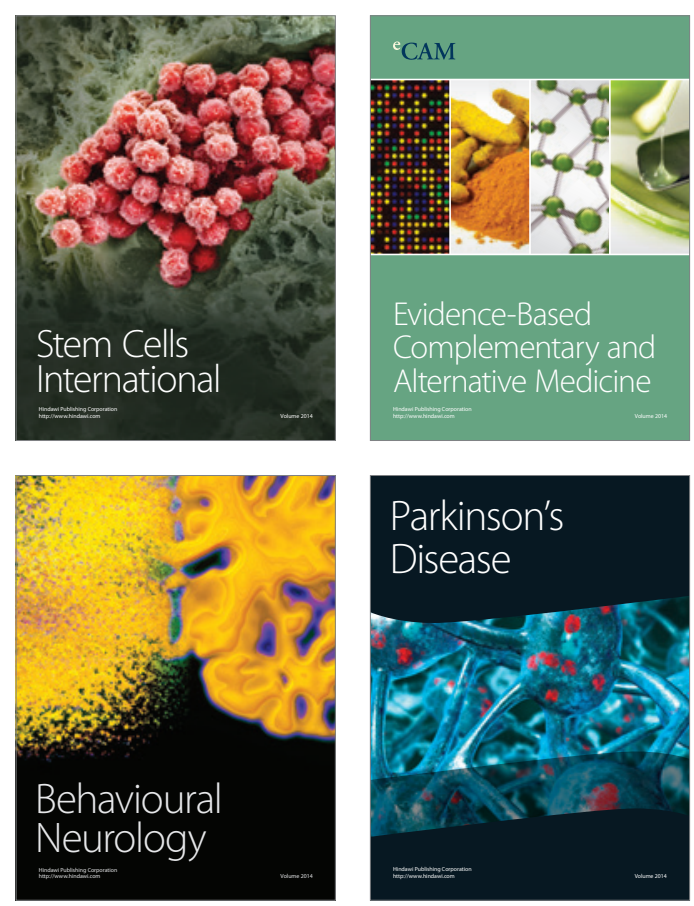

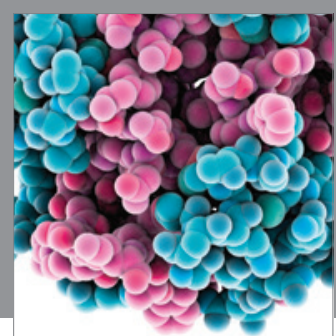

Journal of
Diabetes Research

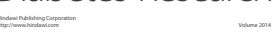

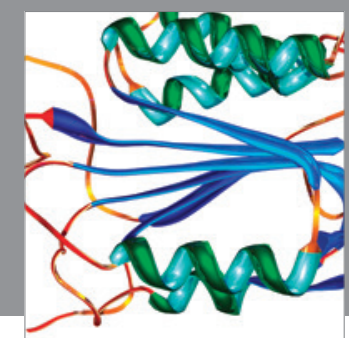

Disease Markers
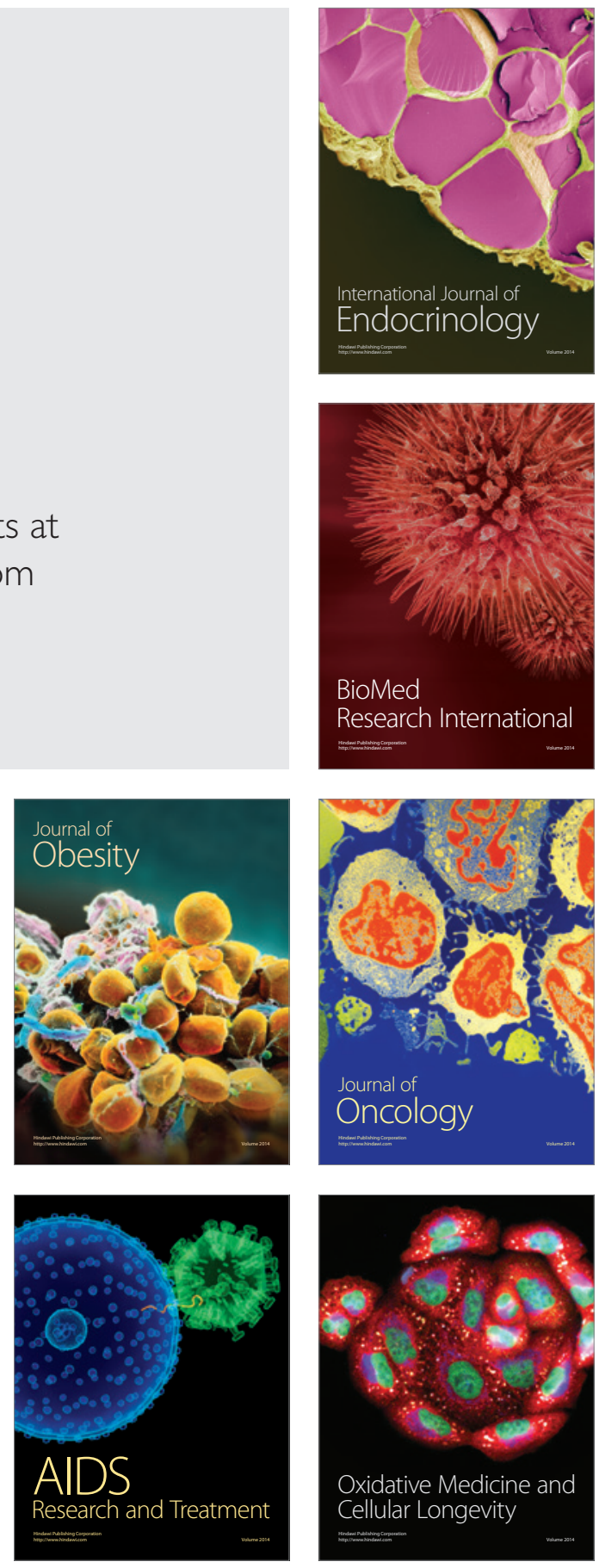